\title{
Relic gravitational waves and their detection
}

\author{
Wen Zhao and Yang Zhang \\ Astrophysics Center, University of Science and Technology of China, Hefei, Anhui, China \\ (Received 26 March 2006; revised manuscript received 18 May 2006; published 2 August 2006)
}

\begin{abstract}
As strong evidence for inflation, relic gravitational waves (RGW) have been extensively studied. Although they have not been detected yet, some constraints have been achieved by observations. Future experiments for RGW detection are mainly of two kinds: CMB experiments and laser interferometers. In this paper, we study these current constraints and the detective abilities of future experiments. We calculate the strength of RGW $\Omega_{g}(k)$ using two methods: the analytic method and the numerical method, by solving the inflationary flow equations. By the first method, we obtain a bound $\Omega_{g}<3.89 \times 10^{-16}$ at $\nu=0.1 \mathrm{~Hz}$, where we have used the current constraints on the scalar spectral index and the tensor-scalar ratio; furthermore, we have taken into account the redshift-suppression effect, the accelerating expansion effect, and the neutrino damping effect on RGW. But the analytic expression of $\Omega_{g}(k)$ depends on specific inflationary models and does not apply well for the waves with very high frequencies. The numerical method is more precise for the waves with high frequencies. It gives a bound $\Omega_{g}<8.62 \times 10^{-14}$, which is independent of the inflationary parameters, and applies to any single-field slow-roll inflationary model. After considering the current constraints on the inflationary parameters, this bound becomes $\Omega_{g}<2 \times$ $10^{-17}$. These two methods give consistent conclusions: the current constraints on RGW from LIGO, big bang nucleosynthesis, and pulsar timing are too loose to give any constraint for the single-field inflationary models, and the constraints from WMAP are relatively tighter. Future laser interferometers are more effective for detecting RGW with the smaller tensor-scalar ratio, but the CMB experiments are more effective for detecting the waves with the larger ratio. These detection methods are complementary to each other for the detection of RGW.
\end{abstract}

DOI: 10.1103/PhysRevD.74.043503

PACS numbers: 98.80.-k, 98.80.Es, 04.30.-w, 04.62.+v

\section{INTRODUCTION}

In the past, a number of observations on the cosmic microwave background (CMB) radiation power spectra [1-3] and on the large-scale structure (LSS) [4] have supported inflation as the good phenomenological model to describe the evolution of the universe at a very early stage, which naturally gives rise to the origin of primordial fluctuations with a nearly scale-invariant and Gaussian spectrum. In addition to the primordial density perturbations, inflationary models also predict a stochastic background of relic gravitational waves (RGW), which are the tensor perturbations. The detection of such a background would provide incontrovertible evidence that inflation actually occurred and would also set strong constraints on the dynamics of inflation [5].

There are mainly two kinds of experiments to detect RGW at different frequencies. For RGW of very low frequencies, $\nu \simeq\left(10^{-17}-10^{-15}\right) \mathrm{Hz}$, one can observe them by detecting the power spectrum of CMB $B$ polarizations [6]. Now, the three-year results of WMAP [2] have not yet found the evidence of RGW. The experiment of the Planck satellite [7] with higher sensitivity to polarization is scheduled for launch in 2007, and the Clover [8] and CMBPol [9] projects with much higher sensitivities than Planck are also under development. For RGW of high frequencies, $\nu \simeq\left(10^{-4}-10^{4}\right) \mathrm{Hz}$, another kind of experiment applies, i.e. the laser interferometer detectors, including the current TAMA [10], VIRGO [11], LIGO [12,13], and the future LISA [14], ASTROD [15], BBO [16], and DECIGO [17].
Besides these two kinds, other methods have also been used to constrain the strength of RGW, such as the timing studies on the millisecond pulsars, which can constrain the amplitude of gravitational waves by studying the signal residuals of the millisecond pulses [18]. This method is sensitive to the waves with frequencies from $10^{-9}-10^{-7} \mathrm{~Hz}$. The observational results of big bang nucleosynthesis $(\mathrm{BBN})$ can also constrain the strength of RGW [19-21] at all frequencies. Although RGW have not been found yet, some constraints on them have already been obtained by these experiments and observations.

The purpose of this paper is to study the various modifications on the power spectrum of RGW and to examine the constraints on the power spectrum from the experiments. On the spectrum, we will consider modifications due to such important effects as the redshift-suppression effect, the accelerating expansion effect, and the neutrino damping effect. Two methods will be applied in this study: the analytic method and the numerical method, by solving the inflationary flow equations. After considering all these damping effects, we will get an analytic formula of the strength of RGW, which, as a function of the wave number $k$, depends on the scalar spectral index $n_{s}$ and the tensorscalar ratio $r$. By taking into account the current observational constraints on $n_{s}$ and $r$, we will obtain an upper limit on the strength of RGW $\Omega_{g}<3.89 \times 10^{-16}$ at $\nu=$ $0.1 \mathrm{~Hz}$. From the $r-\Omega_{g}$ plane, we find that the BBO experiments can detect RGW if $r>8.3 \times 10^{-3}$, which is more sensitive than the Planck satellite, but less sensitive than 
Clover and CMBPol. But the would-be ultimate DECIGO can detect RGW if $r>6.8 \times 10^{-6}$, which is much more sensitive than those CMB experiments. In this analytic method, $\Omega_{g}$ depends on the ratio $r$, an undetermined parameter, whose value varies for the various, specific inflationary models. Furthermore, the approximate power law of the primordial power spectrum may also yield a fairly large error.

To overcome these shortcomings, we move on to the numerical method. Because the RGW depends sensitively on the inflationary stage, during which it is generated, and there are a number of inflationary models, the numerical method is used by which a great many realizations are produced that represents the respective inflationary models. The inflationary flow equations are applied to numerically calculate RGW, whereby an upper limit $\Omega_{g}<$ $8.62 \times 10^{-14}$ is obtained for any slow-roll, single-scalarfield inflationary model, independent of any inflationary parameters. Taking into account the current observed constraints on $n_{s}, r$, and $\alpha$ (the running of the scalar spectral index), we arrive at a much tighter limit, $\Omega_{g}<2 \times 10^{-17}$, which is beyond the sensitivity range of BBO. By numerically generating $10^{7}$ realizations, we find that all of them satisfy the current constraints on $\Omega_{g}$ from LIGO, from pulsar timing, and from BBN, but only nearly $0.05 \%$ of them satisfy the current constraints on $n_{s}, \alpha$, and $r$. From the resulting $r-\Omega_{g}$ plane, one finds the DECIGO, if put into running, will be effective for detecting RGW with smaller $r$, but the CMB experiments, such as Planck, Clover, and CMBPol, are more effective for detecting RGW with larger $r$. They are complementary to each other for RGW detection. Our result from this numerical investigation applies only to the single-field inflationary models with the chosen initial conditions of Hubble slow-roll parameters.

The organization of this paper is as follows. Section II gives a simple review on RGW and their evolution equation. In Sec. III, an analytic expression of the strength of RGW will be obtained with three damping factors being included, presenting the modifications due to the mentioned effects. In Sec. IV, the strength of RGW is numerically calculated by solving the inflationary flow equations. Finally, Sec. V is devoted to conclusions.

\section{RELIC GRAVITATIONAL WAVES AND THEIR EVOLUTION EQUATION}

Incorporating the perturbations to the spatially flat Friedmann-Robertson-Walker (FRW) spacetime, the metric is

$$
d s^{2}=a(\tau)^{2}\left[d \tau^{2}-\left(\delta_{i j}+h_{i j}\right) d x^{i} d x^{j}\right],
$$

where $a$ is the scale factor of the universe and $\tau$ is the conformal time, which relates to the cosmic time by $a d \tau \equiv$ $d t$. The perturbation of spacetime $h_{i j}$ is a $3 \times 3$ symmetric matrix. The gravitational wave field is the tensorial portion of $h_{i j}$, which is transverse-traceless $\partial_{i} h^{i j}=0, \delta^{i j} h_{i j}=0$. Since the RGW is very weak, $\left|h_{i j}\right| \ll 1$, one just needs to study the linearized evolution equation:

$$
\partial_{\mu}\left(\sqrt{-g} \partial^{\mu} h_{i j}\right)=16 \pi G a^{2}(\tau) \Pi_{i j}
$$

where $\Pi_{i j}$ is the tensor part of the anisotropy stress, satisfying $\Pi_{i i}=0$, and $\partial_{i} \Pi_{i j}=0$. It couples to $h_{i j}$ as an external source. In the cosmic setting, $\Pi_{i j}$ can be generated by the free-streaming relativistic particles [22,23], the cosmic magnetic particles [24], etc. It is convenient to Fourier transform these quantities as follows:

$$
\begin{gathered}
h_{i j}(\tau, \mathbf{x})=\sum_{\lambda} \sqrt{16 \pi G} \int \frac{d \mathbf{k}}{(2 \pi)^{3 / 2}} \epsilon_{i j}^{(\lambda)}(\mathbf{k}) h_{\mathbf{k}}^{\lambda}(\tau) e^{i \mathbf{k} \mathbf{x}}, \\
\Pi_{i j}(\tau, \mathbf{x})=\sum_{\lambda} \sqrt{16 \pi G} \int \frac{d \mathbf{k}}{(2 \pi)^{3 / 2}} \epsilon_{i j}^{(\lambda)}(\mathbf{k}) \Pi_{\mathbf{k}}^{\lambda}(\tau) e^{i \mathbf{k} \mathbf{x}},
\end{gathered}
$$

where the index $\lambda="+$ " or " $\times$ " labels the two polarization states of the gravitational waves. The polarization tensors $\boldsymbol{\epsilon}_{i j}^{(\lambda)}$ are symmetric, transverse-traceless $k^{i} \epsilon_{i j}^{(\lambda)}(\mathbf{k})=0, \delta^{i j} \epsilon_{i j}^{(\lambda)}(\mathbf{k})=0$, and satisfy the conditions $\epsilon^{(\lambda) i j}(\mathbf{k}) \epsilon_{i j}^{\left(\lambda^{\prime}\right)}(\mathbf{k})=2 \delta_{\lambda \lambda^{\prime}}$ and $\epsilon_{i j}^{(\lambda)}(-\mathbf{k})=\epsilon_{i j}^{(\lambda)}(\mathbf{k})$. Since the RGW is assumed to be isotropic and each polarization state is the same, $h_{\mathbf{k}}^{(\lambda)}(\tau)$ is denoted by $h_{k}(\tau)$, and $\Pi_{\mathbf{k}}^{(\lambda)}(\tau)$ by $\Pi_{k}(\tau)$, where $k=|\mathbf{k}|$ is the wave number related to the frequency by $\nu \equiv k / 2 \pi$ (the present scale factor is set as $a_{0}=1$ ). Then, Eq. (2) can be rewritten as

$$
\ddot{h}_{k}+2 \frac{\dot{a}}{a} \dot{h}_{k}+k^{2} h_{k}=16 \pi G a^{2}(\tau) \Pi_{k}(\tau),
$$

where the overdot denotes a conformal time derivative $d / d \tau$. Although the interactions between gravitational waves and other matter are very weak, in many cases, the source $\Pi_{k}$ in Eq. (5) is negligible and the evolution of RGW only depends on the scale factor and its time derivative; however, in this paper we include this source term, so that the damping from neutrino free-streaming is properly taken care of.

\section{THE ANALYTIC POWER SPECTRUM OF RGW}

\section{A. The primordial power spectrum of RGW}

Inflationary expansion, as an attractive idea to describe the very early universe, has received strong support from the observations of CMB anisotropies and from studies of the large-scale distribution of the galaxy. Here we will consider only the single-field models. In the context of slow-roll inflationary models, the observables depend on three slow-roll parameters [25]: 


$$
\begin{gathered}
\epsilon_{V} \equiv \frac{M_{\mathrm{Pl}}^{2}}{2}\left(\frac{V^{\prime}}{V}\right)^{2}, \quad \eta_{V} \equiv M_{\mathrm{Pl}}^{2}\left(\frac{V^{\prime \prime}}{V}\right), \\
\xi_{V} \equiv M_{\mathrm{Pl}}^{4}\left(\frac{V^{\prime} V^{\prime \prime \prime}}{V^{2}}\right),
\end{gathered}
$$

where $M_{\mathrm{Pl}} \equiv(8 \pi G)^{-1 / 2}=m_{\mathrm{Pl}} / \sqrt{8} \pi$ is the reduced Planck energy, $V(\phi)$ is the inflationary potential, and the prime denotes derivatives with respect to the field $\phi$. Here, $\epsilon_{V}$ quantifies the "steepness" of the slope of the potential, $\eta_{V}$ quantifies the "curvature" of the potential, and $\xi_{V}$ quantifies the "jerk." All three of these parameters must be smaller than 1 for inflation to occur. One of the important predictions of the inflationary models is the primordial scalar perturbation power spectrum, which is nearly Gaussian and nearly scale invariant. This spectrum is written in the form

$$
P_{S}(k)=P_{S}\left(k_{0}\right)\left(\frac{k}{k_{0}}\right)^{n_{s}\left(k_{0}\right)-1+(1 / 2) \alpha \ln \left(k / k_{0}\right)},
$$

where $n_{s}$ is the scalar spectral index, $\alpha \equiv d n_{s} / d \ln k$ is its running, and $k_{0}$ is some pivot wave number. In this paper, $k_{0}=0.05 \mathrm{Mpc}^{-1}$ is taken. The observations of WMAP give $P_{S}\left(k_{0}\right) \simeq 2.95 \times 10^{-9} A\left(k_{0}\right)$ and $A\left(k_{0}\right)=0.9 \pm 0.1$ [1]. Another major prediction of inflationary models is the existence of RGW. The primordial power spectrum of RGW is defined by

$$
P_{T}(k) \equiv \frac{32 G k^{3}}{\pi} h_{k}^{+} h_{k}
$$

where $h_{k}$ is the solution of Eq. (5). This spectrum can also be put in a simple form,

$$
P_{T}(k)=P_{T}\left(k_{0}\right)\left(\frac{k}{k_{0}}\right)^{n_{t}\left(k_{0}\right)+(1 / 2) \alpha_{t} \ln \left(k / k_{0}\right)},
$$

where $n_{t}(k)$ is the tensor spectral index, and $\alpha_{t} \equiv$ $d n_{t} / d \ln k$ is its running. In the single-field inflationary models, a standard slow-roll analysis gives the following relations:

$$
\begin{gathered}
n_{t}=-\frac{r}{8}, \quad \alpha_{t}=\frac{r}{8}\left[\left(n_{s}-1\right)+\frac{r}{8}\right], \\
r=\frac{8}{3}\left(1-n_{s}\right)+\frac{16}{3} \eta_{V},
\end{gathered}
$$

where $r(k) \equiv P_{T}(k) / P_{S}(k)$ is the so-called tensor-scalar ratio. These formulas relate the tensorial parameters $n_{t}$ and $\alpha_{t}$ to the scalar parameters $n_{s}$ and $r$; the latter are accessible to the observations of CMB and LSS. As shown in Eq. (10), the relation between $r$ and $n_{s}$ involves the slowroll parameter $\eta_{V}$, depending on the specific inflationary potential. Inserting these into Eq. (9), one gets

$$
\begin{aligned}
P_{T}(k)= & P_{S}\left(k_{0}\right) \times r \\
& \times\left(\frac{k}{k_{0}}\right)^{-(r / 8)+(r / 16)\left[\left(n_{s}-1\right)+(r / 8)\right] \ln \left(k / k_{0}\right)} .
\end{aligned}
$$

In general, the tensor-scalar ratio $r$ may vary with the wave number $k$. Here and in the following sections we will take the value of $r$ at $k=k_{0}$, i.e. $r \equiv r\left(k_{0}\right)$. Now the primordial spectrum of RGW only depends on the parameters $n_{s}$ and $r$. The recent constraints by the observations of three-year WMAP, SDSS, SNIa, and galaxy clustering [26] are

$$
\begin{gathered}
n_{s}=0.965 \pm 0.012 \quad(68 \% \text { C.L. }) \text {, } \\
r<0.22 \quad(95 \% \text { C.L. }) .
\end{gathered}
$$

The strength of the gravitational waves can be characterized by the gravitational waves' energy spectrum

$$
\Omega_{g}(k)=\frac{1}{\rho_{c}} \frac{d \rho_{g}}{d \ln k},
$$

where $\rho_{c}=3 H_{0}^{2} / 8 \pi G$ is the critical density and $H_{0}=$ $100 \mathrm{hm} \mathrm{s}^{-1} \mathrm{Mpc}^{-1}$ is the present Hubble constant (the value $h=0.72$ is taken throughout this paper). $\Omega_{g}$ can be related to the primordial power spectrum by the formula $[23,27]$

$$
\Omega_{g}(k)=\frac{1}{12 H_{0}^{2}} k^{2} P_{T}(k) \mathcal{T}^{2}(k),
$$

where the transfer function $\mathcal{T}(k)$ will take into account the various damping effects mentioned earlier, and will be discussed below.

\section{B. Damping effects}

Here, three kinds of damping effects will be addressed. First, we only consider the redshift-suppression effect caused by the overall expansions of the universe. So, temporarily, we drop the anisotropy stress term $\Pi_{k}(\tau)$ in Eq. (5) due to neutrino free-streaming,

$$
\ddot{h}_{k}+2 \frac{\dot{a}}{a} \dot{h}_{k}+k^{2} h_{k}=0 \text {. }
$$

This equation of RGW only depends on the behavior of the scale factor $a(\tau)$. It has been known that, during the expansion of the universe, the mode function $h_{k}(\tau)$ of the gravitational waves behaves differently in two regimes: far outside the horizon $(k \ll a H)$ and far inside the horizon $(k \gg a H)$. When waves are far outside the horizon, the amplitude of $h_{k}$ stays constant, and when inside the horizon, the amplitude is damping with the expansion of the universe,

$$
h_{k} \propto \frac{1}{a(\tau)} .
$$

By numerically integrating Eq. (16), this effect can be approximately described by a transfer function [28]

$$
t_{1}(k)=\frac{3 j_{1}\left(k \tau_{0}\right)}{k \tau_{0}} \sqrt{1.0+1.36\left(\frac{k}{k_{\text {eq }}}\right)+2.50\left(\frac{k}{k_{\text {eq }}}\right)^{2}},
$$

where $k_{\text {eq }}=0.073 \Omega_{m} h^{2} \mathrm{Mpc}^{-1}$ is the wave number cor- 
responding to the Hubble radius at the time that matter and radiation have equal energy densities, and $\tau_{0}=1.41 \times$ $10^{4} \mathrm{Mpc}$ is the present conformal time. It is obvious that this factor $t_{1}(k)$ is oscillating with the wave number $k$ caused by the Bessel function $j_{1}\left(k \tau_{0}\right)$. In practice, one is usually interested in the amplitude of RGW, as the quick oscillations are of no importance. For the waves with $k \tau_{0} \gg 1$, this factor can be written as

$$
t_{1}(k)=\frac{3}{\left(k \tau_{0}\right)^{2}} \sqrt{1.0+1.36\left(\frac{k}{k_{\mathrm{eq}}}\right)+2.50\left(\frac{k}{k_{\mathrm{eq}}}\right)^{2}} .
$$

It should be noticed that the above transfer function (19) does not include the effect of the recent accelerating expansion of the universe, which has been strongly supported by observations. The spectrum of RGW has been studied in specific models for dark energy [29], such as the Chaplyngin gas models and the $X$-fluid model. In Ref. [30], we have presented an analytic solution of $\mathrm{RGW}$ in the $\Lambda \mathrm{CDM}$ universe, and found that the amplitude of the gravitational waves has been modified by the presence of the dark energy during the current expansion. In the range of higher frequencies $\left(\nu \gg 3 \times 10^{-18} \mathrm{~Hz}\right)$ in which we are interested in this paper, the amplitude acquires an overall factor $\Omega_{m} / \Omega_{\Lambda}$ as compared with the decelerating model, where $\Omega_{m}$ and $\Omega_{\Lambda}$ are the present energy densities of matter and the vacuum, respectively. So this effect can be simply described by a damping factor,

$$
t_{2}=\frac{\Omega_{m}}{\Omega_{\Lambda}}
$$

In the standard $\Lambda$ CDM model with $\Omega_{m}=0.27$ and $\Omega_{\Lambda}=$ 0.73 , this effect contributes a damping factor of $t_{2}{ }^{2} \sim$ 0.137 for the strength of RGW in Eq. (15).

The third to be considered is the damping effect of the free-streaming neutrinos [22], i.e. the anisotropic stress $\Pi_{k}$ on the right-hand side of Eq. (5). This effect was first considered by Weinberg in Ref. [22]. This effect is primarily produced by neutrinos when they are decoupled and are free streaming in the universe, especially right after the waves enter the horizon. The overall amplitude of RGW will be reduced by roughly $20 \%$. It has been shown that anisotropy stress can reduce the amplitude for wavelengths that reenter the horizon during the radiation-dominated stage, and the damping factor is only dependent on the fraction $f$ of the free-streaming relativistic particles over the background (critical) energy density in the universe. The effect is less for wavelengths that enter the horizon at later times. A number of works have been done to discuss this effect, and in Ref. [23] the authors found that the effect can be approximately described by a transfer function $t_{3}$ for the waves with frequencies $\nu>10^{-16} \mathrm{~Hz}$ (which reenter the horizon at the radiation-dominant stage),

$$
t_{3}=\frac{15\left(14406 f^{4}-55770 f^{3}+3152975 f^{2}-48118000 f+324135000\right)}{343(15+4 f)(50+4 f)(105+4 f)(180+4 f)} .
$$

When the wave modes $\left(10^{-16} \mathrm{~Hz}<\nu<10^{-10} \mathrm{~Hz}\right)$ reenter the horizon, the temperature in the universe is relatively low $(<1 \mathrm{MeV})$; the neutrino is the only free-streaming relativistic particle. So we choose $f=0.4052$, corresponding to 3 standard neutrino species; the damping factor is $t_{3}=0.80313$. But for the waves with higher frequencies $\left(\nu>10^{-10} \mathrm{~Hz}\right)$, the temperature of the universe is very high when they reenter the horizon, and the value of $f$ is much more uncertain. This is because the detail of how many species of particles are free is not accurately known. Thus, the detection of RGW at this frequency offers the possibility of learning about the free-streaming fraction $f$ in the very early universe. In this paper, we choose $f=$ 0.4052 when the waves in $\nu \in\left[10^{-16}, 10^{-10}\right] \mathrm{Hz}$. And for the waves with $\nu>10^{-10} \mathrm{~Hz}$, at the time when the waves reentered the horizon, the temperature was high and the neutrinos were in thermal equilibrium. So we choose $f=$ 0 , i.e. without free-streaming relativistic particles, which corresponds to $t_{3}=1$. But for the waves with $\nu<$ $10^{-16} \mathrm{~Hz}$, which reenter the horizon during the matterdominated stage, the neutrino density is small and its damping impact can be neglected, so we choose $t_{3}=1$.
That is,

$$
t_{3} \simeq \begin{cases}1, & \nu<10^{-16} \mathrm{~Hz} \\ 0.80313, & 10^{-16} \mathrm{~Hz}<\nu<10^{-10} \mathrm{~Hz}, \\ 1, & \nu>10^{-10} \mathrm{~Hz} .\end{cases}
$$

Therefore, the total transfer function is the combination of these three effects,

$$
\mathcal{T}(k)=t_{1} \times t_{2} \times t_{3}
$$

Among these, $t_{1}$ is dominantly important, which approximately shows the evolution of RGW in the expanding universe. The function $t_{2}$ has relatively smaller damping on RGW, which accounts for the accelerating expansion of the universe quite recently $(z \sim 0.3)$. The value of $\Omega_{g}$ is reduced by nearly an order for this effect. The function of $t_{3}$ has the most uncertainty in this discussion. In the extreme case with $f=0$, one has $t_{3}=1$, i.e. no damping; and in another extreme case with $f=1$, one has $t_{3}=0.35$, the smallest value of $t_{3}$. In the case with $f=0.4052, t_{3}=$ 0.80313 , only contributing a damping factor $t_{3}{ }^{2}=0.645$ for the strength of RGW. 
There are some other possible mechanisms, which might affect the amplitude of the gravitational waves: for example, the QCD transition [31,32], $e^{+} e^{-}$annihilation [31-33], cosmic reheating [30,34], and so on [23]. These could influence the value of the expansion rate $\dot{a} / a$, and therefore affect the strength of RGW. However, these effects are either small (as shown in the literature) in comparison with the effects we have discussed, or there are some uncertainties in their analysis, so these are not considered here.

\section{The upper limit of $\boldsymbol{\Omega}_{g}$ and the sensitivities of future experiments}

Future detectors of RGW are mainly classified into two kinds: one kind is through CMB for very low frequencies, and another is based on laser interferometers for relatively high frequencies. For the waves of very low frequencies, $\nu<10^{-15} \mathrm{~Hz}$, the CMB experiments are sensitive. For instance, the Planck satellite can detect RGW if $r>0.1$ [7], the ground-based experiment Clover can detect the signal if $r>0.005$ [8], and CMBPol can detect it if $r>$ $10^{-3}$ is satisfied [9]. It should be noted that, if $r<1 \times$ $10^{-4}$, the RGW may not be detected by CMB experiments. This is because the CMB $B$ polarizations generated by the cosmic lensing are also very large, and the signals from RGW may be subdominant to the lensing effects [35].

The direct detections of RGW by laser interferometers are sensitive to the waves with high frequencies. For the waves with $\nu>10^{-10} \mathrm{~Hz}$, inserting the formulas (19)(23) with $t_{3}=1$ in Eq. (15), the strength of the gravitational waves becomes

$$
\Omega_{g}(k)=\frac{22.5}{12 H_{0}^{2}} \frac{P_{T}(k)}{\tau_{0}^{4} k_{\mathrm{eq}}^{2}}\left(\frac{\Omega_{m}}{\Omega_{\Lambda}}\right)^{2} \simeq 1.08 \times 10^{-6} P_{T}(k) .
$$

Using the expression of $P_{T}(k)$ in Eq. (11), one gets

$$
\begin{aligned}
\Omega_{g}(k) \simeq & 2.87 \\
& \times 10^{-15} r\left(\frac{k}{k_{0}}\right)^{-(r / 8)+(r / 16)\left[\left(n_{s}-1\right)+(r / 8)\right] \ln \left(k / k_{0}\right)},
\end{aligned}
$$

where $A\left(k_{0}\right)=0.9$. This function depends on the wave number $k$, the tensor-scalar ratio $r$, and the scalar spectral index $n_{s}$.

The advanced LIGO can detect the waves with $\Omega_{g} h^{2}>$ $10^{-9}$ at $\nu \simeq 100 \mathrm{~Hz}$ [13]; the LISA project is expected to detect waves with $\Omega_{g} h^{2}>10^{-11}$ at $\nu \simeq 0.005 \mathrm{~Hz}$ [14]; the ASTROD, a space project sensitive to the waves with frequencies at $\nu \in\left(10^{-5}, 10^{-3}\right) \mathrm{Hz}$ [15], is expected to detect the waves with $\Omega_{g} h^{2}>10^{-15}$ at $\nu \simeq 5 \times$ $10^{-4} \mathrm{~Hz}$. The BBO, another important project, can detect a background RGW with $\Omega_{g}>2.2 \times 10^{-17}$ at $\nu \simeq$ (0.1-1) Hz [16]. The DECIGO project, having a much

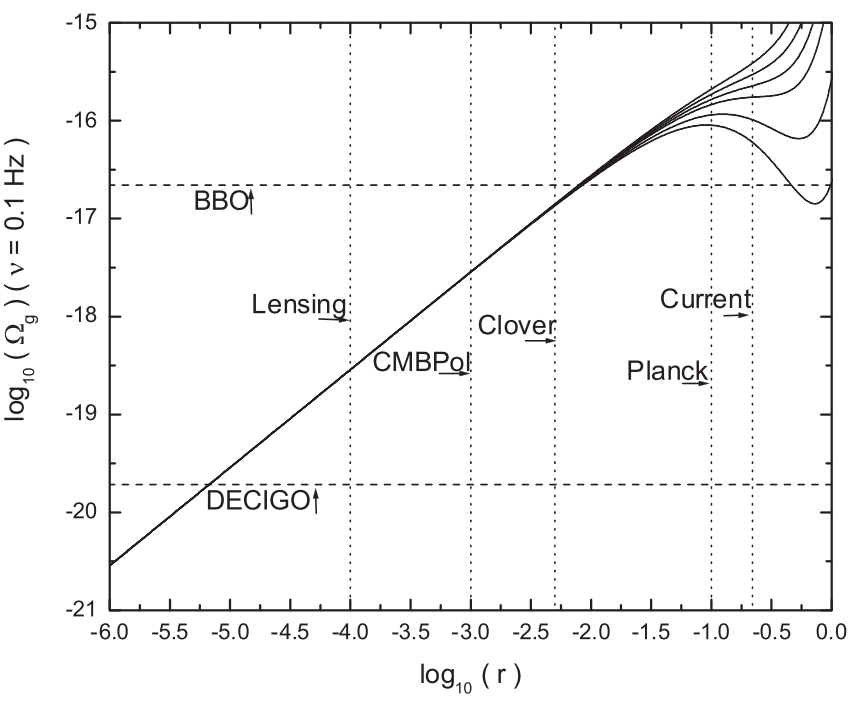

FIG. 1. The strength of RGW at $\nu=0.1 \mathrm{~Hz}$ depends on the slow-roll parameters $n_{s}$ and $r$. This figure shows the results of the analytic approximation in (25). The solid lines from up to down are the curves with $n_{s}=1.00,0.98,0.96,0.94,0.90,0.86$, respectively. The vertical (dotted) lines from right to left are the sensitivity limit curves of current observations, Planck, Clover, CMBPol, and the sensitivity limits of CMB observations, respectively. The horizontal (dashed) lines from up to down are the sensitivity limit curves of BBO and DECIGO, respectively.

higher sensitivity by design, is expected to detect RGW with $\Omega_{g} h^{2}>10^{-20}$ at $\nu \simeq 0.1 \mathrm{~Hz}$ [17].

First, we will estimate the upper limit on the strength of RGW in Eq. (25). Here we assume $n_{s} \leq 1$ and $r<0.22$, which are consistent with the current observations [26]. The formula (25) gives an upper limit of $\Omega_{g}$ at $\nu=0.1 \mathrm{~Hz}$ :

$$
\Omega_{g}<3.89 \times 10^{-16} \text {. }
$$

And this limit is arrived at when $n_{s}=1$ and $r=0.22$. This limit is nearly an order smaller than the result in Ref. [27]. This is because our analysis has taken into account the damping effect of the accelerating expansion of the universe and the running of $n_{t}$ in the primordial spectrum. This limit is in the sensitivity ranges of BBO and DECIGO, but beyond those of LIGO, LISA, and ASTROD. In Fig. 1, we plot the strength of RGW at $\nu=0.1 \mathrm{~Hz}$, as a function of $r$, where several models with different $n_{s}$ are demonstrated. One sees that, when $r<0.01$, the curves of the function $\Omega_{g}$ are almost overlapped for the models of different $n_{s}$, and only depend on the variable $r$. But when $r>$ 0.01 , the models of different $n_{s}$ can be distinguished. For a fixed $r$, a larger $n_{s}$ yields a larger $\Omega_{g}$. This figure also tells us that BBO can detect RGW if $r>8.3 \times 10^{-3}$, so it is more sensitive than the Planck satellite, but less than Clover and CMBPol. It is interesting to notice that DECIGO can detect RGW if $r>6.8 \times 10^{-6}$, which is 
much more sensitive than all the CMB experiments $(r>$ $\left.10^{-4}\right)$.

\section{Predictions of inflationary models}

The strength of RGW in Eq. (25) depends on the values of $n_{s}$ and $r$. Observations have yielded fairly solid constraints on $n_{s}$, but the value of $r$ is still uncertain. The relation between $n_{s}$ and $r$ depends on specific inflationary models, and different models will predict very different $r$. In the following we will discuss several inflationary models, which predict different values of $r$ and yield different $\Omega_{g}$. One may categorize slow-roll models into several classes according to the parameter space spanned by $n_{s}$, $\alpha$, and $r$ [36]. Each class should correspond to specific physical models of inflation. Here we categorize the models according to the curvature of potential $\eta_{V}$ in Eq. (6), as it is the only parameter that enters into the relation (10) between $n_{s}$ and $r$. The classes are defined in the following:

Case A: Negative curvature models $\eta_{V}<0$.

The negative $\eta_{V}$ models often arise from a potential of spontaneous symmetry breaking. One type of popular potential has the form of $V=\Lambda^{4}\left[1-(\phi / \mu)^{p}\right]$, where $p \geq 2$ [36]. This kind of model predicts the red tilt $n_{s}<1$, which is consistent with the observations of three-year WMAP. Also, these models predict fairly small $r$. For the model with $p=2$,

$$
r \simeq 8\left(1-n_{s}\right) e^{-N\left(1-n_{s}\right)},
$$

where $N$ is the number of $e$ folds, taken to be in the range $N \in[40,70]$ to account for the current observations on CMB $[1,2,37]$. Here we choose the value $N=70$. Using the constraint on $n_{s}$ in Eq. (12) yields the constraint $r \in$ [0.014, 0.037]. From Fig. 1, one finds this is beyond the sensitivity range of the Planck satellite, but within the sensitivity ranges of Clover and CMBPol. It is also in the sensitivity ranges of $\mathrm{BBO}$ and DICIGO. In other models with $p>2$, the predicted values of $r$ are much smaller than that of the model with $p=2$.

Case B: Small positive curvature models $0 \leq \eta_{V} \leq$ $2 \epsilon_{V}$.

These models contain as two examples the monomial potentials $V=\Lambda^{4}(\phi / \mu)^{p}$ with $p \geq 2$ for $0<\eta_{V}<2 \epsilon_{V}$ and the exponential potential $V=\Lambda^{4} \exp (\phi / \mu)$ for $\eta_{V}=$ $2 \epsilon_{V}$. In these models, to the first order in slow roll, the scalar index is always red $n_{s}<1$ and the following constraint on $r$ is satisfied:

$$
\frac{8}{3}\left(1-n_{s}\right) \leq r \leq 8\left(1-n_{s}\right) .
$$

Using the constraint on $n_{s}$ in Eq. (12), one finds that $r \in$ $[0.061,0.376]$, which is in the sensitivity ranges of Clover, CMBPol, BBO, and DECIGO. The sensitivity limit of Planck is just in this span, so it may be able to detect the model.

Case C: Intermediate positive curvature models $2 \epsilon_{V}<$ $\eta_{V} \leq 3 \epsilon_{V}$.
The supergravity-motivated hybrid models having a potential of the form $V \simeq \Lambda^{4}\left[1+\alpha \ln (\phi / Q)+\lambda(\phi / \mu)^{4}\right]$, up to one-loop correction, belong to this class. In this case,

$$
n_{s}<1, \quad r>8\left(1-n_{s}\right)
$$

are satisfied. Using the constraint on $n_{s}$ in Eq. (12), one finds that $r>0.184$, which is very close to the current upper limit $r<0.22$. Figure 1 shows that this is in the sensitivity range of the Planck satellite.

Case D: Large positive curvature models $\eta_{V}>3 \epsilon_{V}$.

This class of models has a typical monomial potential similar to case A, but with a plus sign for the term $(\phi / \mu)^{p}$ : $V=\Lambda^{4}\left[1+(\phi / \mu)^{p}\right]$. This enables inflation to occur for a small value of $\phi<m_{\mathrm{Pl}}$. This model predicts a blue tilt of the scalar index $n_{s}>1$, which is in contradiction to the constraint in Eq. (12). But we should note that the observations of three-year WMAP have not ruled out the blue spectrum. If the running of $n_{s}$ with the wave number $k$ is allowed and the contributions of tensor fluctuations are considered, then the best fit of WMAP data suggests that $n_{s}\left(k=0.002 \mathrm{Mpc}^{-1}\right)=1.21_{-0.16}^{+0.13}$ and $\alpha(k=$ $\left.0.002 \mathrm{Mpc}^{-1}\right)=-0.102_{-0.043}^{+0.050}$ [2]. This is a blue spectrum with a negative running. So the determination of the value of $n_{s}$ depends on the more precise observations.

\section{INFLATIONARY FLOW EQUATIONS AND PREDICTIONS FOR RGW}

In the discussions above, the RGW given by the analytic expression (25) depends on the value of the tensor-scalar ratio $r$, which has not yet been determined by observations. Moreover, Eq. (25) is a good approximation only for the waves with wave number around $k \simeq k_{0}\left(\sim 10^{-16} \mathrm{~Hz}\right)$. Therefore, for the high frequency wave with $\nu=0.1 \mathrm{~Hz}$, nearly 15 orders larger than the value of $k_{0}$, this approximate formula may have large errors and is inapplicable. To avoid these flaws, in this section we will employ the technique of the inflationary flow equations to relate $\mathrm{RGW}$ of lower frequencies to that of higher frequencies.

\section{A. Inflationary flow equations}

The inflationary flow equations were first introduced by Hoffman and Turner [38] as a way of generating a large number of slow-roll inflationary models to be compared to the observational data. This method applies to any slowroll, single-scalar-field inflationary models, and relies on defining a set of Hubble slow-roll parameters, which are the derivatives of the Hubble parameter during inflation. The major advantage of this method is that it removes the field from the dynamics, and allows one to study the generic behavior of slow-roll inflation without making detailed assumptions about the underlying particle physics. In this section, we will also use this method to generate a large number of inflationary models, the observables of which are required to be consistent with the current observational constraints at low frequencies. Then we will 
numerically solve the strength of RGW at very high frequencies. In this method the Hubble slow-roll parameters are defined by

$$
\begin{aligned}
\epsilon(\phi) & \equiv \frac{m_{\mathrm{Pl}}^{2}}{4 \pi}\left(\frac{H^{\prime}(\phi)}{H(\phi)}\right)^{2}, \\
\lambda_{l}(\phi) & \equiv\left(\frac{m_{\mathrm{Pl}}^{2}}{4 \pi}\right)^{l} \frac{\left(H^{\prime}\right)^{l-1}}{H^{l}} \frac{d^{(l+1)} H}{d \phi^{(l+1)}} \quad(l \geq 1),
\end{aligned}
$$

where primes are derivatives with respect to the scalar field $\phi$, and $H(\phi)$ is the Hubble parameter as a function of $\phi$, related to the potential $V(\phi)$ by the so-called HamiltonJacobi formula,

$$
\left[H^{\prime}(\phi)\right]^{2}-\frac{12 \pi}{m_{\mathrm{Pl}}^{2}} H^{2}(\phi)=-\frac{32 \pi^{2}}{m_{\mathrm{Pl}}^{4}} V(\phi) .
$$

These Hubble slow-roll parameters satisfy an infinite set of hierarchical equations, called inflationary flow equations:

$$
\begin{gathered}
\frac{d \epsilon}{d N}=\epsilon(\sigma+2 \epsilon) \\
\frac{d \sigma}{d N}=-\epsilon(5 \sigma+12 \epsilon)+2\left(\lambda_{2}\right), \\
\frac{d}{d N} \lambda_{l}=\left[\frac{l-1}{2} \sigma+(l-2) \epsilon\right] \lambda_{l}+\lambda_{l+1} \quad(l \geq 2)
\end{gathered}
$$

where $N$ is the number of $e$ folds of the inflation, and $\sigma \equiv$ $2 \lambda_{1}-4 \epsilon$. There are two families of fixed points of these flow equations: one is that $\epsilon=0, \lambda_{l}=0$ for $l \geq 2$, and $\sigma=$ constant. In Ref. [39], the authors found that, only if $\sigma>0$, this fixed point is stable, i.e. the attractor solution. The other family of fixed points is given by $\epsilon=$ constant, $\sigma=-2 \epsilon, \lambda_{2}=\epsilon^{2}$, and $\lambda_{l}=\epsilon \lambda_{l-1}$ for $l \geq 3$. And later we will show that the second family of fixed points is not stable. The slow-roll parameters tend to run to the attractor with the expansion of the universe, as long as the slow-roll condition $\epsilon<1$ is satisfied. In order to actually solve this infinite series of equations numerically, it must be truncated by setting a sufficiently high slow-roll parameter to zero, i.e. $\lambda_{m+1}=b$, with $b$ being a constant, and $\lambda_{m+2}=0$ for some suitably large $m$. In this section, when numerically solving the inflationary flow equations, we make the truncation of this series at $m=10$, and choose a set of acceptable initial conditions as in Refs. [27,39]:

$$
\begin{gathered}
\left.\epsilon\right|_{i} \in[0,0.8], \\
\left.\sigma\right|_{i} \in[-0.5,0.5], \\
\left.\lambda_{2}\right|_{i} \in[-0.05,0.05], \\
\left.\lambda_{l}\right|_{i} \in\left[-0.025 \times 5^{-l+3}, 0.025 \times 5^{-l+3}\right]
\end{gathered}
$$

where the subscript $\left.\right|_{i}$ denotes the corresponding initial values. This 11-equation set in Eqs. (32)-(34) is an autonomous system [39]. We choose the constant $b \neq 0$, and set the left-hand side of these equations to be zero. Then we find the only real solution for this 11-equation set,

$$
\begin{array}{ll}
\epsilon_{c}=b^{1 / 11}, & \sigma_{c}=-2 b^{1 / 11}, \\
\lambda_{l c}=b^{l / 11} & (2 \leq l \leq 10),
\end{array}
$$

where the subscript $c$ means the fixed point. This is just the second family of fixed points with $\epsilon_{c}=b^{1 / 11}$. In order to study the stability of this fixed point, let us consider the small perturbations, i.e.

$$
\begin{array}{lr}
\epsilon=\epsilon_{c}+\delta \epsilon, & \sigma=\sigma_{c}+\delta \sigma, \\
\lambda_{l}=\lambda_{l c}+\delta \lambda_{l} & (2 \leq l \leq 10) .
\end{array}
$$

Substituting these into Eqs. (32)-(34), one gets the firstorder differential equations

$$
\frac{d}{d N}\left(\begin{array}{c}
\delta \epsilon \\
\delta \sigma \\
\cdot \\
\cdot \\
\delta \lambda_{10}
\end{array}\right)=M\left(\begin{array}{c}
\delta \epsilon \\
\delta \sigma \\
\cdot \\
\cdot \\
\delta \lambda_{10}
\end{array}\right),
$$

where the matrix $M$ depends upon the values of $\epsilon_{c}, \sigma_{c}$, and $\lambda_{l c}, l=2, \ldots, 10$. If this fixed point is stable, at least, it is necessary that the real parts of the eigenvalues of the matrix $M$ are negative [40]. However, no matter what value of $b \neq 0$ we choose, this condition cannot be satisfied. So, this fixed point is not a stable fixed point. If we choose the value $b=0$, this 11 -equation set in Eqs. (32)-(34) has the first kind of fixed point, which is stable only if $\sigma_{c}>0$ is satisfied.

It is obvious that the evolutions of this 11-equation set are different for the conditions with different $b$. Although whether the fixed points are stable or not depends on the value of $b$, in the computation below, we will still do computations for both kinds of initial conditions: one with $b=0$, and the other with

$$
b \in\left[-0.025 \times 5^{-8}, 0.025 \times 5^{-8}\right] .
$$

It turns out that, for these two cases, our calculational results of $\Omega_{g}$ are very similar. So, in the following sections, we will only show the results of the former case of $b=0$.

\section{B. Inflationary parameters and the strength of RGW}

Many observable inflationary parameters can be related to the Hubble slow-roll parameters. Here we are only interested in three observable parameters for the slow-roll inflationary models: the tensor-scalar ratio $r$, the scalar spectral index $n_{s}$, and its running $\alpha$, which can be described as (to the second order in the slow roll) [41]

$$
r \simeq 16 \epsilon[1-c(\sigma+2 \epsilon)],
$$




$$
\begin{gathered}
n_{s} \simeq 1+\sigma-(5-3 c) \epsilon^{2}-\frac{1}{4}(3-5 c) \sigma \epsilon+\frac{1}{2}(3-c) \lambda_{2}, \\
\alpha=-\frac{1}{1-\epsilon} \frac{d n_{s}}{d N},
\end{gathered}
$$

where $c=4(\ln 2+\gamma)-5 \simeq 0.0814514$ (with $\gamma$ the Euler-Mascheroni constant) is a constant. Once the inflationary flow equations in (32)-(34) are numerically solved, the values of these observables are obtained. Since we are interested in the gravitational waves in a very wide frequency range, $\nu \in\left(10^{-16}, 10^{2}\right) \mathrm{Hz}$, the primordial power spectrum in Eq. (9) may not apply properly. Here we return to its definition. In the slow-roll inflationary models, the primordial power spectrum is [42]

$$
P_{T}(k)=\left.\frac{16}{\pi}\left[1-\frac{c+1}{4} \epsilon\right]^{2} \frac{H^{2}}{m_{\mathrm{Pl}}^{2}}\right|_{k=a H},
$$

where $H$ is the Hubble parameter of inflation when the waves exactly cross the horizon with $k=a H$. If ignoring $\epsilon, P_{T}(k)$ depends only on the Hubble parameter $H$, a result for exact de Sitter inflation. The formula (46) can be rewritten as

$$
P_{T}(k)=\left(\frac{4-(c+1) \epsilon}{4-(c+1) \epsilon_{i}}\right)^{2} \frac{H^{2}}{H_{i}^{2}} P_{T}\left(k_{0}\right),
$$

where $\epsilon_{i}$ and $H_{i}$ are the respective values of $\epsilon$ and $H$ when $k_{0}$ exactly crosses the horizon at $a=k_{0} / H_{i}$. As before, the $\mathrm{RGW}$ power spectrum can be related to the scalar one by $P_{T}\left(k_{0}\right)=P_{S}\left(k_{0}\right) r\left(k_{0}\right)$. The value of $H$ is also related to $H_{i}$ by the following:

$$
H(N)=H_{i} \exp \left[-\int_{N_{i}}^{N} \epsilon(n) d n\right],
$$

where $N_{i}$ is the number of $e$ folds if $H=H_{i}$. Inserting Eqs. (47) and (48) into Eq. (15), one gets the strength of RGW,

$$
\begin{aligned}
\Omega_{g}(k)= & 2.21 \times 10^{-10} r\left(\frac{k}{H_{0}}\right)^{2} \mathcal{T}(k)^{2}\left(\frac{4-(c+1) \epsilon}{4-(c+1) \epsilon_{i}}\right)^{2} \\
& \times \exp \left[-2 \int_{N_{i}}^{N} \epsilon(n) d n\right]
\end{aligned}
$$

where $\mathcal{T}(k)$ is the damping factor, and $H_{0}$ is the present Hubble constant. Using the damping factors in Eqs. (19), (20), and (22) and the expression of $r$ in Eq. (43), one finds that, for the waves with $\nu>10^{-10} \mathrm{~Hz}$, the strength of $\mathrm{RGW}$ is

$$
\begin{aligned}
\Omega_{g}(k) \simeq & 4.59 \times 10^{-14}\left[\epsilon_{i}-c\left(\sigma_{i} \epsilon_{i}+2 \epsilon_{i}^{2}\right)\right] \\
& \times\left(\frac{4-(c+1) \epsilon}{4-(c+1) \epsilon_{i}}\right)^{2} \exp \left[-2 \int_{N_{i}}^{N} \epsilon(n) d n\right],
\end{aligned}
$$

which only depends on the Hubble slow-roll parameters $\epsilon$ and $\sigma$. Before we solve it numerically through the infla- tionary flow equations, we first give an estimate of its upper limit. Since $0 \leq \epsilon<1$ is satisfied during inflation, Eq. (50) yields the upper limit when taking $\epsilon=0$,

$$
\Omega_{g}(k)<7.34 \times 10^{-13} \frac{\epsilon_{i}-c\left(\sigma_{i} \epsilon_{i}+2 \epsilon_{i}^{2}\right)}{\left(4-(c+1) \epsilon_{i}\right)^{2}},
$$

which also depends on the values of $\epsilon_{i}$ and $\sigma_{i}$. When $\epsilon_{i}=$ 1 , the right-hand side of this inequality has the maximum value, so one can give a loose upper limit of $\Omega_{g}(k)$,

$$
\Omega_{g}(k)<8.62 \times 10^{-14},
$$

where the approximation $r \simeq 16 \epsilon$ has been used, and the second-order terms of the tensor-scalar ratio $r$ have been omitted. This upper limit holds only if the slow-roll condition is satisfied. Compared with the limit in Eq. (26), this upper limit does not directly depend on the values of $n_{s}$ and $r$. Besides this merit, this limit applies for $\nu>10^{-10} \mathrm{~Hz}$. So, it applies for a wider range of frequencies. It is obvious that this limit is much looser than that in Eq. (26). Notice that the limit (52) is much smaller than the sensitivities of, and therefore cannot be directly detected by, LIGO and LISA, but it is larger than the sensitivities of, and can be detected by, ASTROD, BBO and DECIGO. In writing down the limit (52), $t_{3}=1$ has been used, which is effective for waves with $\nu>10^{-10} \mathrm{~Hz}$. But for waves with $\nu \in$ $\left[10^{-16}, 10^{-10}\right] \mathrm{Hz}$, one should use $t_{3} \simeq 0.80313$, and thus the limit becomes $\Omega_{g}(k)<5.56 \times 10^{-14}$, which is a little tighter than the limit in Eq. (52).

\section{Current constraints on cosmic parameters}

In the discussion above, we know that the values of $n_{s}$, $\alpha, r$, and $\Omega_{g}$ are all directly related to the Hubble slow-roll parameters. Here, we give a review of the current constraints on them. The constraints on the inflationary parameters $n_{s}, \alpha$, and $r$ mainly come from the observations at large scale, including the observations of CMB, LSS, and so on. Here, we call them "large-scale constraints" (LSC). When solving the inflationary flow equations, we will take the initial condition at the time of horizonleaving, i.e. $k_{0}=a H$, where the pivot wave number $k_{0}=$ $0.05 \mathrm{Mpc}^{-1}$ as before.

Now, the WMAP CMB data (1st year) gives [1] $n_{s}=$ $0.93 \pm 0.07, \alpha=-0.047 \pm 0.040$, and the best fit of the WMAPext $+2 \mathrm{dFGRS}$ galaxy survey gives $n_{s}=$ $0.93 \pm 0.03, \alpha=-0.031_{-0.017}^{+0.016}$. A fit using WMAP CMB data and the SDSS galaxy survey gives [43] $n_{s}=0.98 \pm$ $0.02, \alpha=-0.003 \pm 0.010$. Combining the observations of three-year WMAP, SDSS, SNIa, and galaxy clustering [26], one can give the constraints $n_{s}=0.965 \pm 0.012$, $\alpha=-(2.0 \pm 1.2) \times 10^{-2}$, and $r<0.22$. These bounds of $n_{s}$ and $\alpha$ are all at the $68 \%$ confidence level, and those of $r$ are at the $95 \%$ confidence level. These various bounds are consistent with each other, taking into account the corresponding confidence levels. Here, in our calculation 
we will choose the loosest constraints

$n_{s} \in[0.86,1.00], \quad \alpha \in[-0.087,0.007], \quad r<0.22$,

which imply that the primordial scalar spectrum is "red" or scale invariant, and the running of the scalar index is very small, as required by the slow-roll inflationary models.

The constraints on $\Omega_{g}$ mainly come from the observations at small scale. Here, we call them "small-scale constraints" (SSC), which include the tightest constraint from observations of the pulsar timing [18],

$$
\Omega_{g} h^{2}<2 \times 10^{-9}, \quad \nu=1.9 \times 10^{-9} \mathrm{~Hz}
$$

the constraint from the recent observations of LIGO [12],

$$
\Omega_{g}<8.4 \times 10^{-4}, \quad 69 \mathrm{~Hz}<\nu<156 \mathrm{~Hz} ;
$$

and the constraint from the observations of $\mathrm{BBN}[19,20]$,

$$
w_{g} h^{2}<8.9 \times 10^{-6},
$$

where $w_{g} \equiv \int \Omega_{g}(\nu) d \ln \nu$. Comparing with the constraints (26) and (52), it is fair to say that the current $\mathrm{SSC}$ are too loose to give any constraint on the single-field inflationary models. This result will also be checked in the following numerical calculation.

\section{The distribution of the realizations}

In this subsection, we will present a numerical program to solve the inflationary flow equations (32)-(34) for a number of models, where each initial condition randomly chosen within the constraints of (35)-(38) represents a model.

First, we want to study how tight the LSC of Eq. (53) and the SSC of Eqs. (54)-(56) are, as constraints, on the inflationary models. We have produced $10^{7}$ realizations of inflationary models. It turns out that all these realizations satisfy the SSC, which attests to the conclusion before: the current SSC are too loose to give any actual constraint on the single-field inflationary models. On the other hand, among these $10^{7}$ realizations, only 5523 of them ( $\sim 0.05 \%$ ) satisfy the LSC of Eq. (53). So this constraint is tighter for the inflationary models. In the following, we will mainly discuss the distribution of these 5523 realizations.

During the numerical calculations, the inflation can end in one of the following two ways. One is that $\epsilon<1$ is violated in the process of computing; then the inflation automatically stops. A number of inflationary models are of this class, such as the polynomial "large-field" models and the "small-field" polynomial potentials [36]. The other way is by an abrupt termination, perhaps from intervention of an auxiliary field as in hybrid inflation. The linear potentials and the exponential potentials also belong to this class [36]. Here we choose the abrupt stop at $N=$

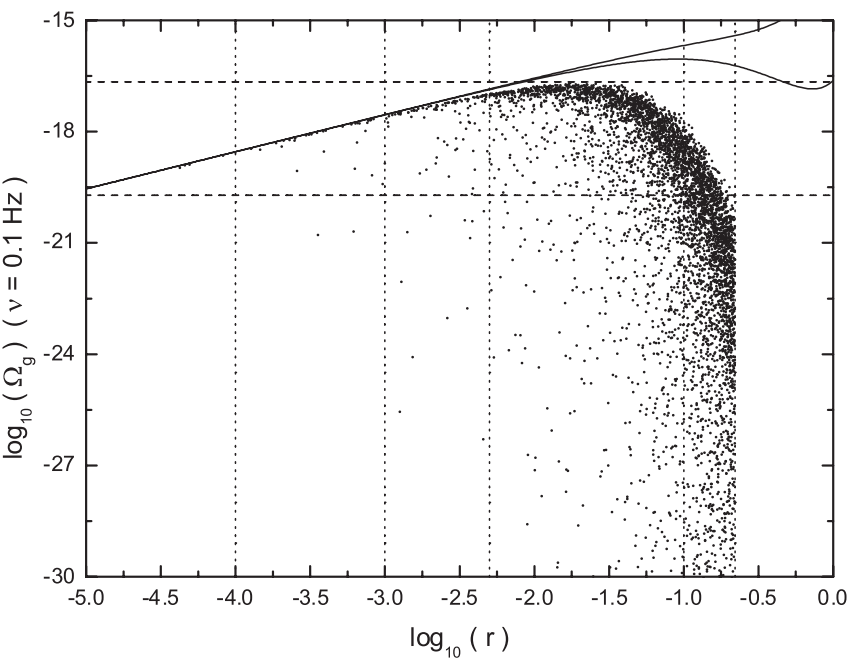

FIG. 2. The distribution of the 5509 realizations in the $r-\Omega_{g}$ plane. The solid lines from up to down are the analytic curves with $n_{s}=1.00$ and $n_{s}=0.86$, respectively. The vertical (dotted) lines and the horizontal (dashed) lines have the exact same meanings as those in Fig. 1.

70 in computation. We have found that, among these 5523 realizations, only 14 of them stop the inflation in the first way; all others do so in the second way. This fact is consistent with previous works $[27,39]$. In the following we will discuss these two kinds of realizations separately.

First, we discuss the 5509 realizations that exit inflation by abrupt termination. They also satisfy both the large- and small-scale constraints in Eqs. (53)-(56). These models can inflate at least $70 e$ folds. In Fig. 2, we plot them in the $r-\Omega_{g}$ plane, which shows the following characters:

(a) For a fixed $r$, the distribution of $\Omega_{g}$ is very scattered, especially in the region with large $r$. For example, for a fixed $r=0.22$, the values of $\Omega_{g}$ are distributed in a broad range, $\Omega_{g} \in\left(10^{-45}, 10^{-20}\right)$;

(b) For each fixed $r$, the values of $\Omega_{g}$ have an upper limit, and the small region just below this limit tends to contain most of the realizations;

(c) For each fixed $r$, the values of the upper limit $\Omega_{g}$ from our numerical result are smaller than the analytic results of Eq. (25), especially in the region $r>$ 0.01 ;

(d) At $r \simeq 0.03$, the strength of RGW can attain the maximum value $\Omega_{g} \simeq 2 \times 10^{-17}$, which is more than an order of magnitude smaller than the analytic result of Eq. (26). And this is beyond the sensitivity ranges of LIGO, LISA, ASTROD, and BBO.

(e) Most of the realizations tend to concentrate in the region with large values of $r$, and the larger $r$ is, the denser the distributions of the realizations are. More than $90 \%$ of realizations are in the region of $r>$ 0.01 . This phenomenon of distribution may be due to our specific choice of the initial conditions in Eqs. (35) $-(38)$. 
Among these 5509 realizations, $50.21 \%$ fall into the sensitivity region of the Planck satellite, $97.11 \%$ fall into that of Clover, $99.29 \%$ fall into that of CMBPol, and $42.91 \%$ fall into the sensitivity region of DECIGO. In comparison with the CMB observations, much less realizations are in the sensitivity regions of laser interferometer detectors. But, DECIGO can detect RGW with $r$ being much smaller than $10^{-4}$, which is beyond the sensitivities of the CMB experiments. This conclusion is the same as the analytic results in Sec. III. Therefore, the CMB experiments and the laser interferometers are complementary to each other for RGW detection.

Now let us look at the 14 realizations that satisfy all the constraints in Eqs. (53)-(56), but end the inflation before the $e$ folds $N=70$ arrive. We found, for these realizations, the values of $e$ folds are all in the region of $N \in[40,70]$, which is consistent with current observations and theoretic predictions [36]. In Fig. 3, we plot them in the $r-\Omega_{g}$ plane. This figure shows an interesting feature: a larger $r$ corresponds to a smaller $\Omega_{g}$, which is also consistent with the distribution of realizations in Fig. 2. Among these realizations, $35.71 \%$ fall into the sensitivity region of the Planck satellite, $100 \%$ fall into the sensitivity regions of Clover and CMBPol, and $64.29 \%$ fall into the sensitivity region of DECIGO. These results are also consistent with the distribution of the previous 5509 realizations.

It should be mentioned that, in our numerical calculation, the initial conditions have been chosen randomly for the Hubble slow-roll parameters in the regions (35)-(38). It is not clear which one is closer to the actual situation of

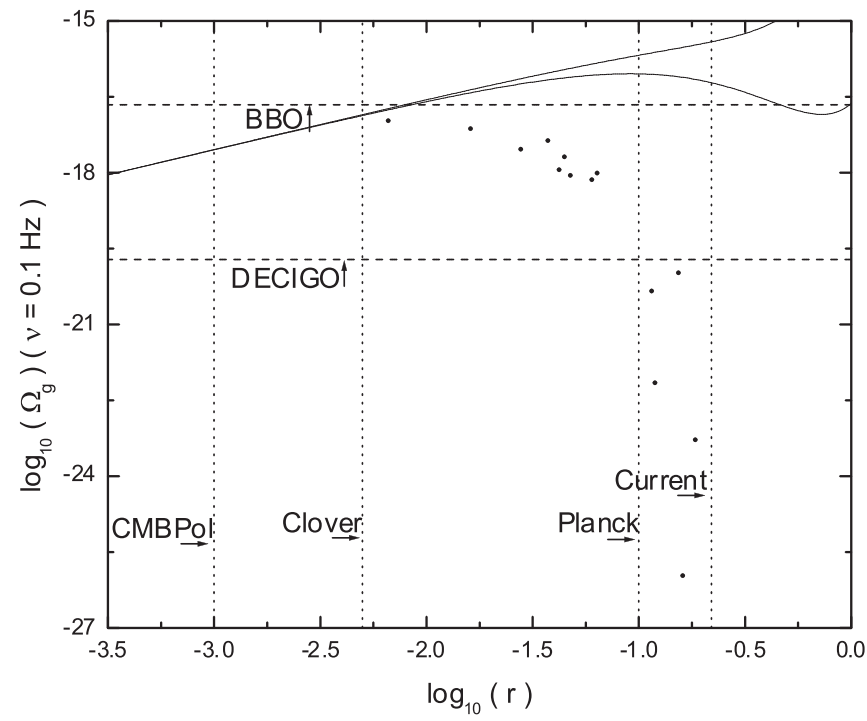

FIG. 3. The distribution of the 14 realizations in the $r-\Omega_{g}$ plane. The solid lines from up to down are the analytic curves with $n_{s}=1.00$ and $n_{s}=0.86$, respectively. The vertical (dotted) lines from right to left are the sensitivity limit curves of current observations, Planck, Clover, and CMBPol, respectively. The horizontal (dashed) lines from up to down are the sensitivity limit curves of BBO and DECIGO, respectively. the inflationary process in the early universe. However, given the very broad range of initial conditions for the Hubble slow-roll parameters, these large samples of $10^{7}$ realizations may exhaust the reasonable reservoir of inflationary models driven by the single scalar field.

\section{CONCLUSION}

Relic gravitational waves are regarded as strong evidence for the inflationary models, which are directly related to the energy scale of inflation. Although up until now people have not observed RGW, a lot of constraints have been achieved on them. These constraints include two kinds: one is the LSC, which is mainly from the CMB observations, especially the recent WMAP results. This can constrain RGW at very low frequencies $\nu \in$ $\left(10^{-17}, 10^{-15}\right) \mathrm{Hz}$. The other is the SSC, which includes the constraints from LIGO, BBN, and pulsar timing, and is sensitive to the waves with high frequencies. A number of experiments are under development for RGW detection, which are also mainly of two kinds: the CMB experiments, including Planck, Clover, CMBPol, and others; and the laser interferometers, including advanced LIGO, LISA, ASTROD, BBO, DECIGO, and so on. The latter ones are sensitive to the waves with $\nu \in\left(10^{-4}, 10^{4}\right) \mathrm{Hz}$.

In this paper, we have calculated the strength of RGW, studied how tight the current constraints are on RGW, and investigated the detective abilities of future experiments. When calculating the values of $\Omega_{g}(k)$, we have used two methods: the analytic method and the numerical method. The former method simply shows the dependent relation of $\Omega_{g}(k)$ on the inflationary parameters $n_{s}$ and $r$. After considering the current constraints on these parameters, we have given an upper limit $\Omega_{g}<3.89 \times 10^{-16}$, where we have included the redshift-suppression effect, the accelerating expansion effect, and the neutrino damping effect of RGW. This limit is in the sensitivity ranges of BBO and DECIGO, but beyond those of advanced LIGO, LISA, and ASTROD. In the numerical method, we calculated the values of $\Omega_{g}(k)$ by solving the inflationary flow equations, which is more precise for RGW in the high frequency range, and also obtained an upper limit $\Omega_{g}<8.62 \times$ $10^{-14}$, which is independent of the inflationary parameters and applies to any single-field slow-roll inflationary model. After considering the constraints on $n_{s}, \alpha$, and $r$, this bound becomes $\Omega_{g}<2 \times 10^{-17}$, which is beyond the sensitivity limit of BBO.

The results from these two methods suggest the following consistent conclusions: the current constraints on RGW from LIGO, BBN, and pulsar timing are too loose to give any constraint on the single-field inflationary models, and the constraints from CMB and LSS are relatively tighter. The future laser interferometer DECIGO is more effective for detecting RGW with smaller $r$, but the CMB experiments, such as Planck, Clover, and CMBPol, are more effective for detecting waves with larger $r$. They are com- 
plementary to each other for RGW detection. The laser interferometers, such as the advanced LIGO, LISA, and ASTROD, have little chance to find the signal of RGW, if the single-field inflationary model is held.

A final remark should be made; that is, all conclusions on RGW and their detection constraints arrived at in this paper are pertinent only for single-scalar-field models for inflation. RGW generated from other models of inflation need to be analyzed separately.

\section{ACKNOWLEDGMENTS}

We thank S. Chongchitnan for helpful discussions. W. Zhao's work has been partially supported by Graduate Student Research Funding from USTC. Y. Zhang's research has been supported by the Chinese NSF Grant No. 10173008, NKBRSF Grant No. G19990754, and by SRFDP.
[1] C. L. Bennett et al., Astrophys. J. Suppl. Ser. 148, 1 (2003); D. N. Spergel et al., Astrophys. J. Suppl. Ser. 148, 175 (2003).

[2] G. Hinshaw et al., astro-ph/0603451; L. Page et al., astroph/0603450; D. N. Spergel et al., astro-ph/0603449.

[3] E. M. Leitch et al., Astrophys. J. 624, 10 (2005); C. J. MacTavish et al., astro-ph/0507503; J.L. Sievers et al., astro-ph/0509203; D. Barkats et al., Astrophys. J. 619, L127 (2005).

[4] S. Cole et al., Mon. Not. R. Astron. Soc. 362, 505 (2005); M. Tegmark et al., Astrophys. J. 606, 702 (2004); D. J. Eisenstein et al., Astrophys. J. 633, 560 (2005).

[5] A. Starobinsky, JETP Lett. 30, 682 (1979); S. Sasaki, Prog. Theor. Phys. 76, 1036 (1986); V.F. Mukhanov, H. A. Feldman, and R. H. Brandenberger, Phys. Rep. 215, 203 (1992); P. J. E. Peebles, Principles of Physical Cosmology (Princeton University Press, Princeton, NJ, 1993); J. A. Peacock, Cosmological Physics (Cambridge University Press, Cambridge, England, 1999); D. H. Lyth and A. Riotto, Phys. Rep. 314, 1 (1999).

[6] U. Seljak and M. Zaldarriaga, Phys. Rev. Lett. 78, 2054 (1997); M. Kamionkowski, A. Kosowsky, and A. Stebbins, Phys. Rev. Lett. 78, 2058 (1997); J. R. Pritchard and M. Kamionkowski, Ann. Phys. (N.Y.) 318, 2 (2005); W. Zhao and Y. Zhang, astro-ph/0508345.

[7] http://www.rssd.esa.int/index.php?project=Planck.

[8] A. C. Taylor et al., astro-ph/0407148.

[9] http://universe.nasa.gov/program/inflation.html; L. Verde, H. Peiris, and R. Jimenez, J. Cosmol. Astropart. Phys. 01 (2006) 019.

[10] http://tamago.mtk.nao.ac.jp/.

[11] http://wwwcascina.virgo.infn.it/.

[12] B. Abbott et al. (LIGO Scientific Collaboration), Phys. Rev. Lett. 95, 221101 (2005).

[13] http://www.ligo.caltech.edu/advLIGO.

[14] http://lisa.nasa.gov/.

[15] W. T. Ni, S. Shiomi, and A. C. Liao, Classical Quantum Gravity 21, S641 (2004).

[16] http://universe.nasa.gov/program/bbo.html; V. Corbin and N. J. Cornish, Classical Quantum Gravity 23, 2435 (2006).

[17] N. Seto, S. Kawamura, and T. Nakamura, Phys. Rev. Lett. 87, 221103 (2001).

[18] S. Detweiler, Astrophys. J. 234, 1100 (1979); S. E. Thorsett and R. J. Dewey, Phys. Rev. D 53, 3468 (1996); A. N. Lommen, astro-ph/0208572.
[19] B. Allen, gr-qc/9604033; R. H. Cyburt, J. Ellis, B. D. Fields, and K.A. Olive, Phys. Rev. D 67, 103521 (2003); R. H. Cyburt, B. D. Fields, K. A. Olive, and E. Skillman, Astropart. Phys. 23, 313 (2005); T. L. Smith, E. Pierpaoli, and M. Kamionkowski, Phys. Rev. Lett. 97, 021301 (2006).

[20] M. Maggiore, Phys. Rep. 331, 283 (2000).

[21] E. W. Kolb and M. Turner, The Early Universe (AddisonWesley Publishing Company, Reading, MA, 1990).

[22] S. Weinberg, Phys. Rev. D 69, 023503 (2004); D. A. Dicus and W. W. Repko, Phys. Rev. D 72, 088302 (2005).

[23] L. A. Boyle and P. J. Steinhardt, astro-ph/0512014.

[24] C. G. Tsagas and J. D. Barrow, Classical Quantum Gravity 14, 2539 (1997); C. G. Tsagas and R. Maartens, Phys. Rev. D 61, 083519 (2000).

[25] A. R. Liddle and D.H. Lyth, Phys. Lett. B 291, 391 (1992); Phys. Rep. 231, 1 (1993).

[26] U. Seljak, A. Slosar, and P. McDonald, astro-ph/0604335.

[27] T. L. Smith, M. Kamionkowski, and A. Cooray, Phys. Rev. D 73, 023504 (2006); S. Chongchitnan and G. Efstathiou, Phys. Rev. D 73, 083511 (2006); T. L. Smith, H. V. Peiris, and A. Cooray, Phys. Rev. D 73, 123503 (2006).

[28] M. S. Turner, M. White, and J. E. Lidsey, Phys. Rev. D 48, 4613 (1993).

[29] J. C. Fabris, S. V. B. Goncalves, and M. S. dos Santos, Gen. Relativ. Gravit. 36, 2559 (2004); M. S. Santos, S. V. B. Goncalves, J.C. Fabris, and E. M. de Gouveia Dal Pino, gr-qc/0504032.

[30] Y. Zhang, Y.F. Yuan, W. Zhao, and Y. T. Chen, Classical Quantum Gravity 22, 1383 (2005); Y. Zhang, W. Zhao, Y.F. Yuan, and T. Y. Xia, Chin. Phys. Lett. 20, 1871 (2005); Y. Zhang, X.Z. Er, T. Y. Xia, W. Zhao, and H. X. Miao, Classical Quantum Gravity 23, 3783 (2006).

[31] B. Kampfer, Ann. Phys. (Berlin) 9, 605 (2000); D. J. Schwarz, Ann. Phys. (Berlin) 12, 220 (2003); T. Schaefer, hep-ph/0509068.

[32] Y. Watanabe and E. Komatsu, Phys. Rev. D 73, 123515 (2006).

[33] D. J. Schwarz, Mod. Phys. Lett. A 13, 2771 (1998).

[34] L. P. Grishchuk, Zh. Eksp. Teor. Fiz. 67, 825 (1974); Ann. N.Y. Acad. Sci. 302, 439 (1977); L. P. Grishchuk, V. M. Lipunov, K. A. Postnov, M.E. Prokhorov, and B.S. Sathyaprakash, Phys. Usp. 44, 1 (2001).

[35] M. Zaldarriaga and U. Seljak, Phys. Rev. D 58, 023003 (1998); M. Kesden, A. Cooray, and M. Kamionkowski, 
Phys. Rev. Lett. 89, 011304 (2002); L. Knox and Y.S. Song, Phys. Rev. Lett. 89, 011303 (2002); W. Hu, M. M. Hedman, and M. Zaldarriaga, Phys. Rev. D 67, 043004 (2003).

[36] S. Dodelson, W. H. Kinney, and E. W. Kolb, Phys. Rev. D 56, 3207 (1997); W. H. Kinney, Phys. Rev. D 58, 123506 (1998); H. V. Peiris et al., Astrophys. J. Suppl. Ser. 148, 213 (2003).

[37] L. Alabidi and D. Lyth, J. Cosmol. Astropart. Phys. 05 (2006) 016; astro-ph/0603539.

[38] M. B. Hoffman and M. S. Turner, Phys. Rev. D 64, 023506 (2001); W. H. Kinney, Phys. Rev. D 66, 083508 (2002); A. R. Liddle, Phys. Rev. D 68, 103504 (2003); S.
Chongchitnan and G. Efstathiou, Phys. Rev. D 72, 083520 (2005).

[39] W. H. Kinney, Phys. Rev. D 66, 083508 (2002); R. Easther and W. H. Kinney, Phys. Rev. D 67, 043511 (2003).

[40] E. J. Copeland, A. R. Liddle, and D. Wands, Phys. Rev. D 57, 4686 (1998); B. Gumjudpai, T. Naskar, M. Sami, and S. Tsujikawa, J. Cosmol. Astropart. Phys. 06 (2005) 007.

[41] A. R. Liddle, P. Parsons, and J. D. Barrow, Phys. Rev. D 50, 7222 (1994).

[42] E. D. Stewart and D. H. Lyth, Phys. Lett. B 302, 171 (1993).

[43] U. Seljak et al., Phys. Rev. D 71, 103515 (2005). 